\title{
Answering Questions on COVID-19 in Real-Time
}

\author{
Jinhyuk Lee Sean S. Yi Minbyul Jeong Mujeen Sung \\ Wonjin Yoon Yonghwa Choi Miyoung Ko Jaewoo Kang \\ Korea University \\ \{jinhyuk_lee, seanswyi, minbyuljeong, mujeensung\}@korea.ac.kr \\ \{wjyoon, yonghwachoi, miyoungko, kangj\}akorea.ac.kr
}

\begin{abstract}
The recent outbreak of the novel coronavirus is wreaking havoc on the world and researchers are struggling to effectively combat it. One reason why the fight is difficult is due to the lack of information and knowledge. In this work, we outline our effort to contribute to shrinking this knowledge vacuum by creating COVIDASK, ${ }^{1}$ a question answering (QA) system that combines biomedical text mining and QA techniques to provide answers to questions in real-time. Our system also leverages information retrieval (IR) approaches to provide entity-level answers that are complementary to QA models. Evaluation of COVIDAsK is carried out by using a manually created dataset called COVID-19 Questions which is based on information from various sources, including the CDC and the WHO. We hope our system will be able to aid researchers in their search for knowledge and information not only for COVID-19, but for future pandemics as well.
\end{abstract}

\section{Introduction}

The most recent pandemic to affect humankind is the coronavirus disease 2019 (COVID-19), which has infected nearly 36 million people worldwide as of Oct 9th, $2020^{2}$ and has led researchers and scientists to scramble to find a solution. Despite such efforts being made and experimental results being released, finding a viable treatment or vaccine for COVID-19 still seems far off.

One of the biggest hurdles to this process is the lack of knowledge regarding COVID-19 and the difficulty of finding relevant and reliable information in a timely fashion. Taking these difficulties into consideration, creating a real-time question answering (QA) system would be able to greatly

\footnotetext{
${ }^{1}$ https://covidask.korea.ac.kr

${ }^{2}$ https://www.who.int/emergencies/diseases/novelcoronavirus-2019
}

aid the efforts of researchers to effectively combat the current pandemic.

Recently, QA models have made significant developments in terms of both performance and throughput. Such improvements can be attributed to the introduction of large-scale QA datasets (Hermann et al., 2015; Rajpurkar et al., 2016) and deep learning models (Seo et al., 2016; Devlin et al., 2019), while recent trends also consider the efficiency of such models (Seo et al., 2019; Dhingra et al., 2020). The development of QA models has also benefited other domains. For example, biomedical QA (Tsatsaronis et al., 2012) has seen many advances due to neural QA models (Lee et al., 2020b; Yoon et al., 2019).

Despite such progress, creating a QA model specific to COVID-19 poses several challenges. The first challenge is that there are almost no QA datasets that are tailored specifically to COVID-19 with a few recent exceptions (Möller et al., 2020). This virtually means that models will have to be evaluated in a zero-shot setting. We take the approach of using a real-time QA model (Seo et al., 2019) and evaluate its transferability from existing QA datasets to a COVID-19 dataset that we coin as the COVID-19 Questions dataset. COVID19 Questions is created by using known facts and experimental results of COVID-19.

The second challenge is the incorporation of traditional biomedical text mining tools into existing QA models. In order to address this, we shift our focus to a key feature used in biomedical text mining: biomedical named entities. Named entities provide important information in text, and this is especially so in the biomedical domain. Taking this into account, we use BioBERT-based models (Kim et al., 2019; Sung et al., 2020) to extract and normalize biomedical named entities found in documents that contain answers. This approach would allow researchers to easily navigate named entities that 
are linked to their respective Concept Unique IDs (CUIs) such as Medical Subject Headings (MeSH). To improve the stability of our approach, we incorporate a biomedical entity search engine (Lee et al., 2016) that provides relevant named entities to entity-level queries.

The contributions of our paper are three-fold. First, we present COVIDAsK for real-time QA on coronaviruses to aid researchers and scientists in effectively navigating resources; second, we incorporate various techniques from traditional biomedical text mining (e.g., biomedical named entity linking (NEL)) to enhance the usability of our model; and third, we evaluate COVIDASK on our COVID-19 Questions dataset and publicly release the source code of COVIDASK and COVID-19 Questions for future work. ${ }^{3}$

\section{System Desiderata}

We first specify the overall goals of our system since QA is very broad and has many details to consider (e.g., types of questions, types of answers, etc.). In this section, we detail how we take such desiderata into consideration, and in Section 3 we elaborate on how we adapted COVIDASK to address each desideratum.

Format of Questions We are mainly interested in providing answers that are in English and in a natural language format. Natural language questions can be divided into two types: interrogative sentences (e.g., "Where did COVID-19 originate from?") and short, keyword-based queries (e.g. "COVID-19 origin"). We cover all types of natural language questions regardless of their format, as they all reflect the need for information.

Format of Answers We restrict the format of answers to be contiguous n-grams within a corpus, often referred to as a "span." Although there are models that are able to generate answers that are not restricted to the given corpus (Raffel et al., 2019), the majority of models are based on "answer span extraction" (i.e., finding specific start and end indices of an answer within the given text) (Seo et al., 2016; Devlin et al., 2019). This approach makes modeling easier while maintaining effectiveness.

However, many research questions raised by researchers and scientists often require entire documents as answers rather than a simple answer span (e.g., questions regarding entire experiments or

\footnotetext{
${ }^{3}$ https://github.com/dmis-lab/covidAsk
}

analyses regarding a certain topic). This is a more typical goal of information retrieval (IR) rather than QA. While QA models implicitly perform IR as answers are extracted from documents - especially in the case of open-domain question answering (Chen et al., 2017) - the two have been treated differently in terms of evaluation and modeling and therefore cannot be put on the same pedestal. We mainly focus on evaluating COVIDAsK in a QA fashion, but also carry out IR-style evaluation via the Text Retrieval Conference COVID-19 (TREC-COVID) Challenge (Roberts et al., 2020; Voorhees et al., 2020). ${ }^{4}$

Source of Knowledge Many QA models that use unstructured text are often given a ground-truth document or paragraph that contains an answer to each question. However, it is more realistic to retrieve a relevant document first and then find an answer rather than being provided the document. This type of Retrieve \& Read approach, popularized by Chen et al. (2017), has been termed open-domain $Q A$ since answers are provided directly from $5 \mathrm{M}$ Wikipedia documents that are not restricted to any specific domain. ${ }^{5}$ We use the COVID-19 Open Research Dataset (CORD-19) (Wang et al., 2020) for a domain-specific corpus provided in unstructured text. Note that although COVIDASK is not an opendomain QA model, we borrow many techniques from open-domain QA since COVIDASK needs to handle a very large amount of text as well.

Recency and Significance An important feature that many QA models do not consider is the recency of information. Recency is important as models that use up-to-date information would be able to provide more relevant results. For instance, when asked "Which drugs are effective for COVID19?," a model that selects an answer from the latest documents would provide more value. Another interesting facet of recency is that the desired behavior of the QA model may change depending on the period of time. For example, past reports on other coronavirus-related diseases like the Middle East respiratory syndrome (MERS) do not explicitly mention COVID-19, but may nevertheless provide important clues or information that may help the understanding of COVID-19. This implies that

\footnotetext{
${ }^{4}$ https://ir.nist.gov/covidSubmit/

${ }^{5}$ The term "open" often refers to both the variety of domains and the large scales of corpora. COVIDASK's knowledge source is focused on a single domain but still uses a very large number of documents.
} 


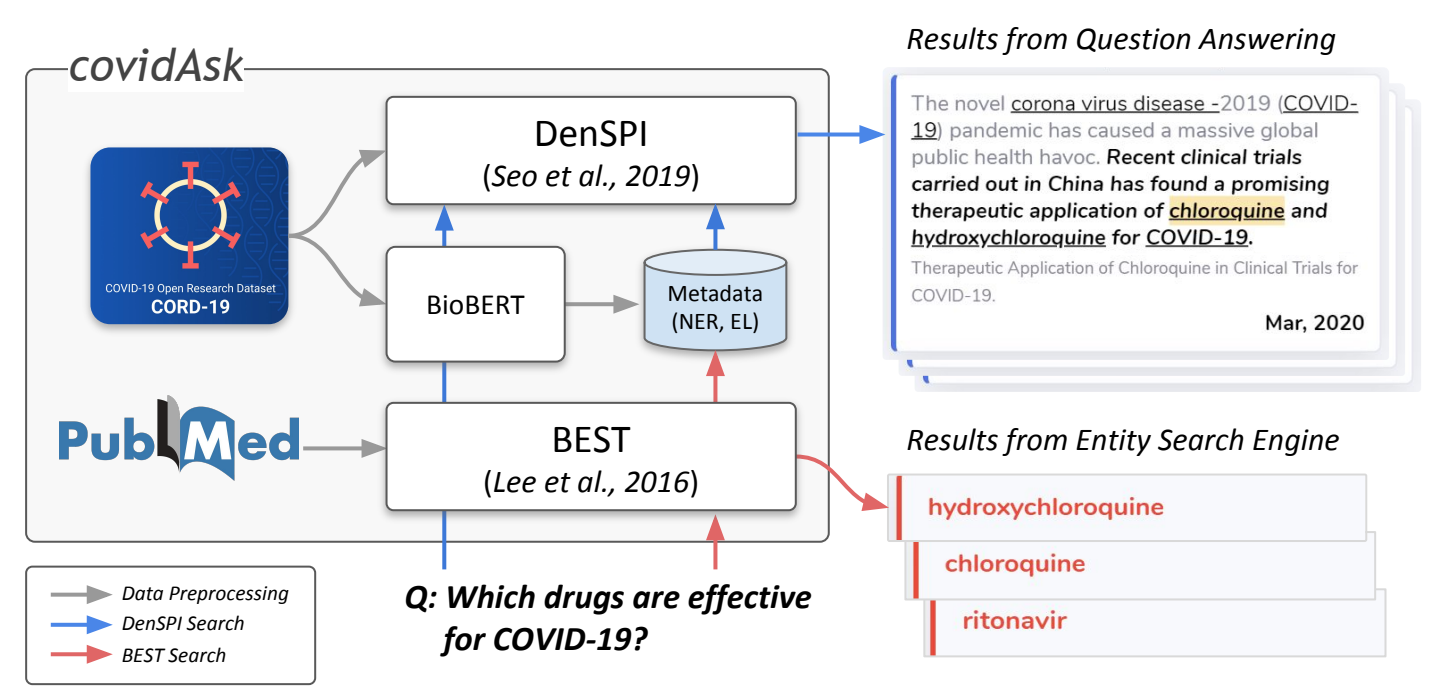

Figure 1: Overview of the COVIDAsK pipeline

when using documents to find answers to questions about COVID-19, it would be appropriate to adopt a more generous stance the older a document is and vice versa.

Another important feature to take into account is the significance of the resources (i.e., how valuable or high quality the resources are). For example, answers that are chosen from research papers that have been peer-reviewed and published at reputable venues would provide more value than those from preprint servers such as medRxiv or bioRxiv. One caveat would be that this may not be applicable to literature related to COVID-19, as review processes for papers are typically lengthy and the current situation calls for the timely release of results. In order to incorporate the concepts of recency and significance into COVIDASK, we leverage the date and impact factor metadata of documents.

User Interaction For each extracted answer, it is essential to also provide the evidence document. This is because it is often necessary to further investigate the retrieved answers by using such evidence documents, as the provided answers themselves may not be enough. To ease such investigation, we extract biomedical named entities in our corpus and link them to their respective CUIs. This allows us to provide detailed descriptions of the named entities including their various synonyms. On top of the named entity recognition (NER) results, we incorporate an entity-level search engine called BEST (Biomedical Entity Search Tool) (Lee et al., 2016) to display important entities relevant to the question.
Latency Latency refers to the delay between inputting a query and receiving an answer. It is an integral aspect of QA models since such models usually deal with large and unstructured sources of knowledge, and therefore speed and efficiency are important. One way that QA models deal with the issue of latency is to retrieve only a small number of documents, thus reducing the search space and focusing only on documents that are likely to be relevant (Chen et al., 2017).

As an alternative, Seo et al. $(2018,2019)$ proposed to pre-index all answer candidate phrases to dense and sparse vectors and perform a maximum inner product search (MIPS) between query vectors and the phrase vectors. We adopt the approach of Seo et al. (2019) because this method allows the model to only have to run through the entire documents once regardless of the number of questions asked, making it more appropriate for real-time QA.

\section{Architecture}

The overall layout of our system is illustrated in Figure 1 and the hosted Web service is shown in Figure 2. We pre-index all phrases in research papers contained in CORD-19 (Wang et al., 2020) and use them to build the DENSPI model (Seo et al., 2019). We also highlight and use biomedical named entities in PubMed ${ }^{6}$ for building BEST (Lee et al., 2016). For given questions, COVIDASK returns two different lists of answers from both DENSPI and BEST.

\footnotetext{
${ }^{6}$ https://www.ncbi.nlm.nih.gov/pubmed/
} 


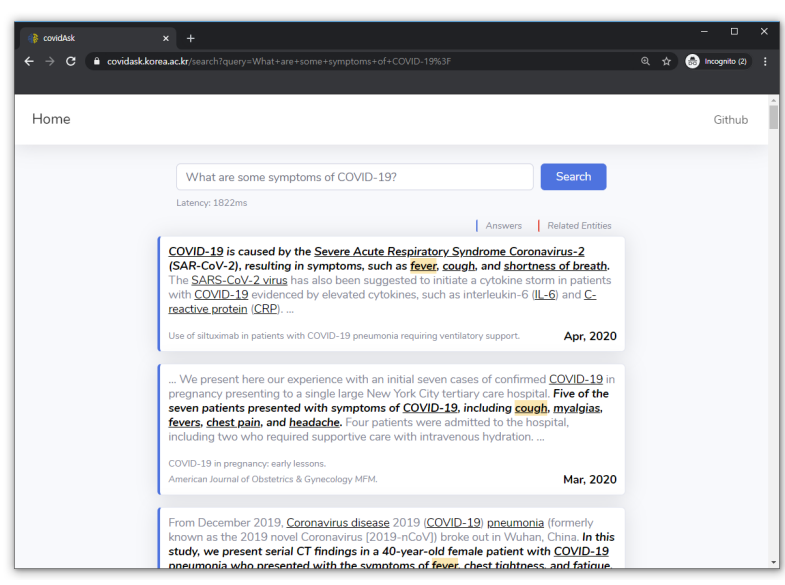

(a) DENSPI results

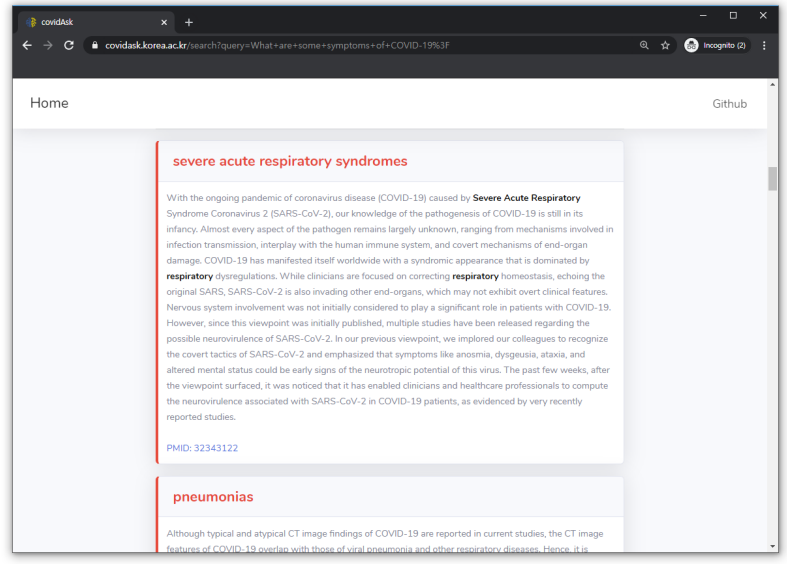

(b) BEST results

Figure 2: Overview of COVIDAsK Web service. (a) and (b) each show the natural question-based and entitybased results of DENSPI and BEST for the question "What are some symptoms of COVID-19?," respectively. BEST results are displayed after DENSPI results.

\subsection{Real-Time Question Answering}

We use DenSPI (Seo et al., 2019) augmented with contextualized sparse representations (SPARC) (Lee et al., 2020a). Among many opendomain QA models, DENSPI has the advantage of being able to provide answers and evidence in real-time (Latency).

First, each phrase vector is supervised with an extractive QA dataset such as SQuAD (Rajpurkar et al., 2016). Next, we encode all answer candidate phrases in a large document corpus into dense and sparse vectors. This essentially enables opendomain QA to be carried out in real-time as retrieving these phrase index vectors is analogous to finding answers and is significantly faster than the conventional method of reading multiple documents whenever a new question is given. Formally speaking, we directly retrieve an answer $\hat{a}$ as:

$$
\hat{a}=\underset{\mathbf{x}_{i: j}^{k}}{\operatorname{argmax}} H_{\mathbf{x}}\left(\mathbf{x}_{i: j}^{k}\right) \cdot H_{\mathbf{q}}(\mathbf{q})
$$

where $H_{\mathbf{x}}$ and $H_{\mathbf{q}}$ are encoding functions for the phrase $\mathbf{x}_{i: j}^{k}$ in the $k$-th document and the question $\mathbf{q}$, respectively. Due to the difficulty of directly building sparse MIPS indices, the sparse vectors are used to re-rank the top 100 phrases. SPARC (Lee et al., 2020a) is used to further enrich the sparse representations of DENSPI with lexical information, which significantly improves performance.

DENSPI provides answers in contiguous $n-$ grams (Format of Answers) for natural language questions (Format of Questions). While the initial version of DENSPI by Seo et al. (2019) is trained on the SQuAD dataset, some work suggests that SQuAD questions are not necessarily "genuine information-seeking questions" in that questions were made with the correct answers in mind (Lee et al., 2019). Hence, we further train DENSPI on the Natural Questions dataset (Kwiatkowski et al., 2019) which contains such information-seeking queries from search engines.

\subsection{Biomedical Named Entity Recognition}

For named entity-based user interaction, we extract and normalize biomedical entities in COVID-19 articles. First, we use BERN (Kim et al., 2019) and BIOSYN (Sung et al., 2020) for biomedical NER and NEL, respectively. Although BERN provides high-quality NER results with BioBERT (Lee et al., 2020b), its entity linking depends on many external tools and dictionaries which are insufficient for high-precision entity linking. BIOSYN, on the other hand, achieves state-of-the-art performance on many biomedical NEL datasets using a synonym marginalization algorithm. We modify BERN to use BIOSYN internally, resulting in a highly accurate NEL system. For each recognized named entity, we provide a link corresponding to its CUI whenever possible. We provide our preprocessed NER results on CORD-19 in a JavaScript Object Notation (JSON) format. ${ }^{7}$

We additionally use BEST (Lee et al., 2016) to provide a list of biomedical named entities that are relevant to the input questions. BEST builds an inverted index that takes biomedical named en-

\footnotetext{
${ }^{7}$ https://github.com/dmis-lab/covidAsk\#data
} 
tities in each PubMed abstract into account and returns entity-level search results. BEST is also more suitable than DENSPI for shorter keywordbased questions (e.g., "What are the symptoms of COVID-19?" vs. "COVID-19 symptoms"). Taking this into consideration, using the two models in concert would allow BEST to provide results that complement those of DENSPI.

\subsection{Incorporating Metadata}

We use of each COVID-19 article's metadata (e.g., date and venue of publication) to help users find more recent and important information (Recency and Significance). In our experiments, we tested re-ranking the results of DENSPI with 1) an impact factor score and 2) the time of publication of each article. However, both showed negative results in terms of our evaluation metric as balancing the importance of metadata and QA results is very difficult. We leave more effective incorporation of the metadata as our future work.

\subsection{User Experience and Interaction}

In Figure 2, we show how COVIDAsK provides search results to users in its Web service. First, users can input any type of question in the search box located at the top. COVIDASK subsequently shows phrase-level answers from CORD-19 using DENSPI (blue tags) and entity-level answers from PubMed articles using BEST (red tags). For DENSPI results, each answer is highlighted in yellow and the sentence containing the answer is in boldface. When users click on a biomedical named entity (underlined), they will be redirected to the web page that contains detailed descriptions from the Comparative Toxicogenomics Database (CTD $)^{8}$ or the National Center for Biotechnology Information's (NCBI's) Taxonomy Database. ${ }^{9}$ Other metadata (e.g., title, date, authors) are also provided whenever available.

\section{Resources}

\subsection{Coronavirus Articles}

COVIDASK mainly uses articles from CORD-19, which is a dataset that contains documents from scientific literature related to the novel coronavirus and other relevant coronaviruses and is updated on a daily basis. ${ }^{10}$ For the experiments, COVI-

\footnotetext{
${ }^{8} \mathrm{http}: / / \mathrm{ctdbase}$. org/

${ }^{9}$ https://www.ncbi.nlm.nih.gov/taxonomy

${ }^{10} \mathrm{https}: / / \mathrm{www}$. semanticscholar.org/cord19/download
}

\begin{tabular}{lccc}
\hline & \multicolumn{3}{c}{ \# of Examples } \\
Dataset & Train & Dev & Test \\
\hline SQuAD & 87,599 & 10,570 & - \\
Natural Questions & 79,168 & 8,757 & - \\
COVID-19 Questions & - & - & 111 \\
TREC-COVID & - & - & 30 \\
\hline
\end{tabular}

Table 1: Statistics of datasets used in COVIDASK

DASK uses the April 17th, 2020 version of CORD19 for the phrase-indexing stage of DENSPI but is updated whenever a newer version is available. We use only the abstract of each article since most key information of the articles is included in the abstract. We also conduct biomedical NER and NEL on the same version of CORD-19.

BEST, on the other hand, utilizes entire PubMed articles including articles on coronaviruses. Consequently, COVIDASK not only performs accurate QA on recent articles related to COVID-19, but also provides entity-level search results obtained from a massive amount of PubMed articles.

\subsection{Question Answering Datasets}

The main challenge of building a QA model in the COVID-19 domain is the lack of training and evaluation data. We mostly rely on existing QA datasets for training and create a separate evaluation dataset using several known facts and experimental results related to COVID-19. The statistics of the training and evaluation sets are listed in Table 1.

Training Set We use two extractive QA datasets for training DENSPI: SQuAD (Rajpurkar et al., 2016) and Natural Questions (Kwiatkowski et al., 2019). We use the preprocessed version of Natural Questions provided by Asai et al. (2019) where long answers are used as the paragraphs for short answers. We limit the maximum number of answer tokens to be 5 in Natural Questions following previous work on the open-domain setup of Natural Questions (Lee et al., 2019).

COVID-19 Questions In order to effectively evaluate COVIDASK, we manually create an evaluation dataset called the COVID-19 Questions dataset. Table 2 shows example questions from each source, and Table 3 displays the basic statistics of COVID19 Questions.

COVID-19 Questions is composed of questions from four different sources: frequent input queries from initial users of COVIDAsK, questions from 


\begin{tabular}{llll}
\hline Source & Type & Question & Answers \\
\hline \multirow{2}{*}{ Query Log } & $\begin{array}{l}\text { Interrogative } \\
\text { Keyword }\end{array}$ & $\begin{array}{l}\text { How long does the virus live outside } \\
\text { COVID-19 vaccines }\end{array}$ & $\begin{array}{l}\text { [three days, 3 hours, ...] } \\
\text { [Chloroquine, Hydroxychloroquine, ... ] }\end{array}$ \\
\hline \multirow{2}{*}{ Kaggle } & $\begin{array}{l}\text { Interrogative } \\
\text { Keyword }\end{array}$ & $\begin{array}{l}\text { What is the recommended length of quarantine? } \\
\text { risk factors of COVID-19 }\end{array}$ & $\begin{array}{l}\text { [three weeks, 14 days, 2-week, ... ] } \\
\text { [hypertension, diabetes, heart disease, ... ] }\end{array}$ \\
\hline \multirow{2}{*}{ CDC \& WHO } & Interrogative & What diseases are caused by coronavirus? & [MERS, SARS, ARDS, COVID-19, ...] \\
& Keyword & medicines or therapies for COVID-19 & [no evidence, no vaccine, no cure, ... ] \\
\hline
\end{tabular}

Table 2: Sample questions from COVID-19 Questions

\begin{tabular}{lccc}
\hline & \multicolumn{3}{c}{ \# of Samples per Type } \\
Source & Interrogative & Keyword & Total \\
\hline Query Log & 4 & 9 & 13 \\
Kaggle & 28 & 28 & 56 \\
CDC \& WHO & 21 & 21 & 42 \\
\hline Total & 53 & 58 & 111 \\
\hline
\end{tabular}

Table 3: Statistics of COVID-19 Questions

the FAQ sections of the Center for Disease Control (CDC) ${ }^{11}$ and the World Health Organization $(\mathrm{WHO})^{12}$ websites, and Kaggle's CORD-19 Challenge tasks page. ${ }^{13}$ We categorized questions from the CDC and the WHO into one category, as the questions from the query log and Kaggle tend to be more formal and academic, whereas those from the CDC and the WHO tend to be more casual (e.g., "What is the basic reproductive number of COVID19?" vs. "What kind of hand sanitizer should I use?"). Regardless of the grammatical details, if the sentence is in a question format we classified it as an interrogative sentence.

The key aspect that we kept in mind when creating COVID-19 Questions was variety. Due to the limited amount of data, it is important that various samples existed in order to effectively determine where COVIDASK performed well and where it did not. In order to test the effect of the question format, we also made sure that each interrogative sentence had a short, keyword-based counterpart and vice versa. We also varied the name used to refer to the novel coronavirus (e.g., COVID-19, SARS-CoV-2, HCoV-19).

TREC-COVID Although COVIDAsK is designed to perform QA, it implicitly performs IR as well since the documents relevant to answers for given questions must first be retrieved. Hence,

\footnotetext{
${ }^{11}$ https://www.cdc.gov/coronavirus/2019-ncov/faq.html

${ }^{12} \mathrm{https} / / / \mathrm{www}$.who.int/csr/disease/coronavirus_ infections/faq_dec12/en/

${ }^{13} \mathrm{https}: / / \mathrm{www} . k a g g l e . c o m / a l l e n-i n s t i t u t e-f o r-a i / C O R D-$ 19-research-challenge/tasks
}

we also evaluate COVIDASK on queries from the TREC-COVID Challenge which is a competition composed of IR-based tasks. The TREC-COVID Challenge is motivated from nine research questions regarding how to use IR in a pandemic situation, and aims to find answers for questions 3 to 8 (Roberts et al., 2020). We participated in the first round which contains 30 topics (i.e., questions) in both interrogative sentence- and short, keywordbased forms.

\section{Experiments}

\subsection{Implementation Details}

For DENSPI, most hyperparameters are identical to the settings in Lee et al. (2020a) except that we stick to the dense-first search strategy in order to increase the diversity of answers. The number of CORD-19 articles (i.e., 37K abstracts) is much smaller than the number of all Wikipedia articles used in open-domain QA, and therefore we use a smaller number of centroids $(1,024$ centroids) for Faiss clustering (Johnson et al., 2019). For BERN and BIOSYN, we modified the original implementations to integrate the two models for biomedical NER and NEL. For BEST, we used the BEST API provided in a Python script. ${ }^{14}$ For the submission of TREC-COVID, we used our COVID19 Questions dataset as a validation set.

Regarding the CORD-19 articles, we mainly used the 2020-04-10 version and additionally created another version of 2020-04-10 that contains only recent articles (i.e., articles published after December, 2019) that we denote as 2020-04-10recent. This way, we effectively reduce the search space while putting more emphasis on recent information. Additionally, we incorporate scores from Covidex (Zhang et al., 2020) in order to obtain better sparse representations for DENSPI.

\footnotetext{
${ }^{14}$ https://github.com/SunkyuKim/BEST_API
} 


\begin{tabular}{|c|c|c|c|c|c|c|c|c|}
\hline Model & Articles & Train & Features & \multicolumn{2}{|c|}{ Interrogative } & \multicolumn{2}{|c|}{ Keyword } & $\mathrm{s} / \mathrm{Q}$ \\
\hline \multirow[t]{2}{*}{ DENSPI + SPARC } & 2020-04-10 & SQuAD & - & 0.3585 & 0.7736 & 0.0862 & 0.4483 & 1.10 \\
\hline & 2020-04-10-recent & SQuAD & Covidex & 0.3208 & 0.7358 & 0.1897 & 0.5172 & 0.87 \\
\hline DENSPI + SPARC & 2020-04-10 & SQuAD + NQ & - & 0.2453 & 0.6038 & 0.1552 & 0.4310 & 0.93 \\
\hline \multirow[t]{2}{*}{ DENSPI (unpublished) } & 2020-04-10-recent & NQ & - & 0.3208 & 0.6415 & 0.1897 & 0.5862 & 1.11 \\
\hline & 2020-06-14-recent & NQ & - & 0.2453 & 0.5283 & 0.2241 & 0.5000 & 0.93 \\
\hline
\end{tabular}

Table 4: Results on COVID-19 Questions

\begin{tabular}{llcccccc}
\hline Team & Run & Train & Features & P@5 & NDCG@ 10 & MAP & Bpref \\
\hline sabir & sab20.1.meta.docs & - & - & 0.7800 & 0.6080 & 0.3128 & 0.4832 \\
UIowaS & UIowaS_Run3 & - & - & 0.6467 & 0.5286 & 0.2625 & 0.4686 \\
covidex & T5R1 & - & - & 0.6467 & 0.5223 & 0.1919 & 0.2838 \\
TU_Vienna & TU_Vienna_TKL_1 & - & - & 0.5133 & 0.4002 & 0.1632 & 0.2545 \\
wistud & wistud_bing & - & - & 0.4467 & 0.3362 & 0.1269 & 0.3110 \\
UB_NLP & UB_NLP_RUN_1 & - & - & 0.3800 & 0.2453 & 0.0574 & 0.2214 \\
\hline \multirow{2}{*}{ KoreaUniversity_DMIS } & dmis-rnd1-run1 & SQuAD & Covidex & 0.5867 & 0.4467 & 0.1202 & 0.2791 \\
& dmis-rnd1-run2 & SQuAD + NQ & Covidex & 0.3867 & 0.3225 & 0.0676 & 0.2339 \\
& dmis-rnd1-run3 & Manual & Covidex & 0.5867 & 0.4649 & 0.1071 & 0.2601 \\
\hline
\end{tabular}

Table 5: Results on TREC-COVID Round 1. For 'Manual' submission, we manually chose better results from dmis-rnd1-run1 and dmis-rnd1-run2 for each query.

Evaluation Metric Many QA works use the Exact Match (EM) and F1 Score (F1) metrics between predicted answers and ground-truth answers, motivated by Rajpurkar et al. (2016). We take a more generous approach to evaluate COVIDASK since our evaluation dataset is relatively small and often has multiple answers for each question. First, we design COVIDAsK to produce a sentence-level answer that contains the predicted phrase-level answer. Next, we use in-sentence EM (EM sent $)$ which is 1 when one of the ground-truth answers is in the predicted answer sentence and 0 otherwise. We also use top- $k \mathrm{EM}_{\text {sent }}\left(\mathrm{EM}_{\text {sent }} @ k\right)$ to evaluate the overall quality of the top- $k$ answers. Our evaluation metric reflects the fact that users of COVIDAsK will need to read the minimal context of answers (i.e., a sentence) regardless of the correctness of the answer itself.

\subsection{Results}

Results on COVID-19 Questions are shown in Table 4. When DENSPI + SPARC is trained on $\mathrm{SQuAD}$, its performance on interrogative questions is generally superior to other models. Although the performance on keyword questions when trained on both SQuAD and NQ improves with 2020-04-10 articles, it does not perform any better with 2020- 04-10-recent articles. Lastly, we include our recent ongoing effort to improve the performance of DENSPI (DENSPI (unpublished)) which is purely trained on Natural Questions. Its performance on keyword questions largely outperforms other models. As shown in the last row, we continue to update COVIDASK whenever more advanced QA models are available.

Table 5 shows the results on TREC-COVID. The results of our submissions (KoreaUniversity_ DMIS) show that COVIDASK is capable of performing IR, although it is not very effective compared to other systems that are fully dedicated to IR rather than QA. This is due to the fact that QA and IR are fundamentally different tasks, as it is also possible for documents unrelated to the question itself to contain correct answers. The discrepancy between evaluation metrics used for QA and IR also imply that the two tasks put emphasis on different aspects. For more details regarding TREC-COVID Round 1 submissions, please refer to the TREC-COVID Round 1 Archive. ${ }^{15}$

\footnotetext{
${ }^{15}$ https://ir.nist.gov/covidSubmit/archive/archiveround1.html
} 


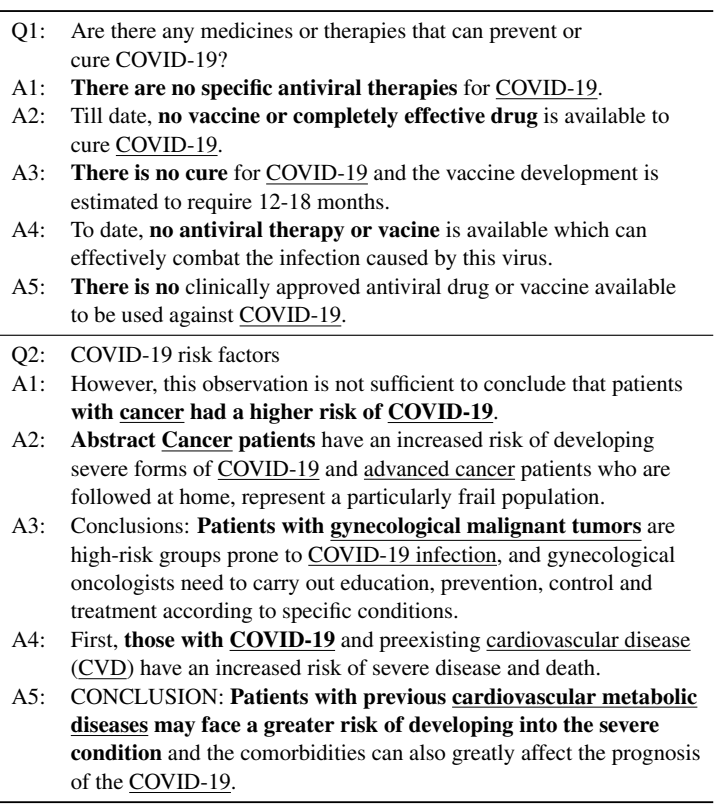

Table 6: Prediction samples of COVIDAsK

\subsection{Qualitative Analysis}

Table 6 displays two example questions each in interrogative sentence and keyword form and the respective sentence-level output prediction of COVIDAsK that had the highest scores (from top to bottom). Within each sentence-level answer, phrase-level answers are in boldface and the linked biomedical entities are underlined. We can see that the usage of existing QA datasets indeed allows COVIDAsK to effectively handle natural questions in both interrogative and keyword forms.

\section{Discussion}

Since the outbreak of COVID-19, there have been various efforts being made from multiple angles in order to handle it. Among those efforts, research focused on building effective QA \& IR systems (Zhang et al., 2020; Das et al., 2020) and dataset curation for such systems (Wei et al., 2020; Gutierrez et al., 2020; Möller et al., 2020) have been especially promising as they can most directly aid researchers in narrowing the knowledge gap.

Taking into consideration the lack of datasets for COVID-19, many IR models for COVID-19 perform in an unsupervised or zero-shot setting. However, unlike IR where unsupervised models and algorithms such as the BM25 (Robertson and Zaragoza, 2009) often produce satisfying results, many QA models rely on a large amount of rich annotations (Seo et al., 2016, 2019; Devlin et al., 2019). While COVIDASK uses QA models trained on SQuAD and Natural Questions and returns zeroshot results on COVID-19 Questions, we believe that COVIDASK could benefit from recent discoveries in unsupervised QA (Lewis et al., 2019), zeroshot QA (Brown et al., 2020), and question generation (Duan et al., 2017; Yang et al., 2017) as well. Further evaluation and fine-tuning could also be conducted using the aforementioned new COVID19 QA datasets.

During the development of COVIDASK, we faced several challenges that are difficult to tackle considering the current state of our model. These include:

- Answerability of questions for a large amount of unstructured text: Although we tried to set a threshold for the scores of answers, the distribution of scores were very inconsistent across different questions (e.g., a top 1 answer with a low score is often correct).

\section{- Leveraging domain adapted datasets:} We tested whether using the BioASQ dataset (Tsatsaronis et al., 2012) could improve the performance of our model, but found that there were no significant improvements compared to those in Table 4.

These challenges are also actively studied in many NLP researches and we hope to tackle them in near future.

\section{Conclusion}

In this work, we presented COVIDASK in an attempt to assist the process of collecting and curating much needed knowledge by providing highly accurate answers to questions in real-time. While COVIDASK is an ongoing endeavor that will be updated accordingly, we provide a cornerstone of constructing a COVID-19 QA system and also make public the source code and evaluation dataset. We plan to further automate and refine our system so that it will be able to be adapted to provide aid in future pandemic situations as well.

\section{Acknowledgments}

This work was supported by the National Research Foundation of Korea (NRF-2020R1A2C3010638, NRF-2016M3A9A7916996) and the Korea Health Industry Development Institute, funded by the Ministry of Health \& Welfare, Republic of Korea (HR20C0021). We thank the members of Korea University and Kyle Lo (Allen Institute for Artificial Intelligence) for their insightful feedback. 


\section{References}

Akari Asai, Kazuma Hashimoto, Hannaneh Hajishirzi, Richard Socher, and Caiming Xiong. 2019. Learning to retrieve reasoning paths over wikipedia graph for question answering. arXiv preprint arXiv:1911.10470.

Tom B Brown, Benjamin Mann, Nick Ryder, Melanie Subbiah, Jared Kaplan, Prafulla Dhariwal, Arvind Neelakantan, Pranav Shyam, Girish Sastry, Amanda Askell, et al. 2020. Language models are few-shot learners. arXiv preprint arXiv:2005.14165.

Danqi Chen, Adam Fisch, Jason Weston, and Antoine Bordes. 2017. Reading wikipedia to answer opendomain questions. In Proceedings of the 55th Annual Meeting of the Association for Computational Linguistics (Volume 1: Long Papers), pages 18701879.

Debasmita Das, Yatin Katyal, Janu Verma, Rajesh Kumar Ranjan, Shashank Dubey, Aakash Deep Singh, Sourojit Bhaduri, and Kushagra Agarwal. 2020. Information retrieval and extraction on covid-19 clinical articles using graph community detection and bio-bert embeddings. https://openreview.net/forum?id=W3Dzaik1ipL.

Jacob Devlin, Ming-Wei Chang, Kenton Lee, and Kristina Toutanova. 2019. Bert: Pre-training of deep bidirectional transformers for language understanding. In Proceedings of the 2019 Conference of the North American Chapter of the Association for Computational Linguistics: Human Language Technologies, Volume 1 (Long and Short Papers), pages 4171-4186.

Bhuwan Dhingra, Manzil Zaheer, Vidhisha Balachandran, Graham Neubig, Ruslan Salakhutdinov, and William W Cohen. 2020. Differentiable reasoning over a virtual knowledge base. In International Conference on Learning Representations.

Nan Duan, Duyu Tang, Peng Chen, and Ming Zhou. 2017. Question generation for question answering. In Proceedings of the 2017 Conference on Empirical Methods in Natural Language Processing, pages 866-874.

Bernal Jimenez Gutierrez, Juncheng Zeng, Dongdong Zhang, Ping Zhang, and Yu Su. 2020. Document classification for covid-19 literature. https://openreview.net/forum?id=SPxaJuM4Hbz.

Karl Moritz Hermann, Tomas Kocisky, Edward Grefenstette, Lasse Espeholt, Will Kay, Mustafa Suleyman, and Phil Blunsom. 2015. Teaching machines to read and comprehend. In Advances in neural information processing systems, pages 1693-1701.

Jeff Johnson, Matthijs Douze, and Hervé Jégou. 2019. Billion-scale similarity search with gpus. IEEE Transactions on Big Data.
Donghyeon Kim, Jinhyuk Lee, Chan Ho So, Hwisang Jeon, Minbyul Jeong, Yonghwa Choi, Wonjin Yoon, Mujeen Sung, , and Jaewoo Kang. 2019. A neural named entity recognition and multi-type normalization tool for biomedical text mining. IEEE Access, 7:73729-73740.

Tom Kwiatkowski, Jennimaria Palomaki, Olivia Redfield, Michael Collins, Ankur Parikh, Chris Alberti, Danielle Epstein, Illia Polosukhin, Jacob Devlin, Kenton Lee, et al. 2019. Natural questions: a benchmark for question answering research. Transactions of the Association for Computational Linguistics, 7:453-466.

Jinhyuk Lee, Minjoon Seo, Hannaneh Hajishirzi, and Jaewoo Kang. 2020a. Contextualized sparse representation for real-time open-domain question answering. In $A C L$.

Jinhyuk Lee, Wonjin Yoon, Sungdong Kim, Donghyeon Kim, Sunkyu Kim, Chan Ho So, and Jaewoo Kang. 2020b. Biobert: a pre-trained biomedical language representation model for biomedical text mining. Bioinformatics, 36(4):1234-1240.

Kenton Lee, Ming-Wei Chang, and Kristina Toutanova. 2019. Latent retrieval for weakly supervised open domain question answering. In Proceedings of the 57th Annual Meeting of the Association for Computational Linguistics, pages 6086-6096.

Sunwon Lee, Donghyeon Kim, Kyubum Lee, Jaehoon Choi, Seongsoon Kim, Minji Jeon, Sangrak Lim, Donghee Choi, Sunkyu Kim, Aik-Choon Tan, et al. 2016. Best: next-generation biomedical entity search tool for knowledge discovery from biomedical literature. PloS one, 11(10).

Patrick Lewis, Ludovic Denoyer, and Sebastian Riedel. 2019. Unsupervised question answering by cloze translation. In $A C L$.

Timo Möller, Anthony Reina, Raghavan Jayakumar, and Malte Pietsch. 2020. Covid-qa: A question \& answer dataset for covid-19. https://openreview.net/forum?id=JENSKEEzsoU

Colin Raffel, Noam Shazeer, Adam Roberts, Katherine Lee, Sharan Narang, Michael Matena, Yanqi Zhou, Wei Li, and Peter J Liu. 2019. Exploring the limits of transfer learning with a unified text-to-text transformer. arXiv preprint arXiv:1910.10683.

Pranav Rajpurkar, Jian Zhang, Konstantin Lopyrev, and Percy Liang. 2016. Squad: 100,000+ questions for machine comprehension of text. In Proceedings of the 2016 Conference on Empirical Methods in Natural Language Processing, pages 2383-2392.

Kirk Roberts, Tasmeer Alam, Steven Bedrick, Dina Demner-Fushman, Kyle Lo, Ian Soboroff, Ellen Voorhees, Lucy Lu Wang, and William R Hersh. 2020. Trec-covid: Rationale and structure of an information retrieval shared task for covid-19. Journal of the American Medical Informatics Association. 
Stephen Robertson and Hugo Zaragoza. 2009. The probabilistic relevance framework: BM25 and beyond. Now Publishers Inc.

Minjoon Seo, Aniruddha Kembhavi, Ali Farhadi, and Hannaneh Hajishirzi. 2016. Bidirectional attention flow for machine comprehension. In International Conference on Learning Representations.

Minjoon Seo, Tom Kwiatkowski, Ankur Parikh, Ali Farhadi, and Hannaneh Hajishirzi. 2018. Phraseindexed question answering: A new challenge for scalable document comprehension. In Proceedings of the 2018 Conference on Empirical Methods in Natural Language Processing, pages 559-564.

Minjoon Seo, Jinhyuk Lee, Tom Kwiatkowski, Ankur Parikh, Ali Farhadi, and Hannaneh Hajishirzi. 2019. Real-time open-domain question answering with dense-sparse phrase index. In Proceedings of the 57th Annual Meeting of the Association for Computational Linguistics, pages 4430-4441.

Mujeen Sung, Hwisang Jeon, Jinhyuk Lee, and Jaewoo Kang. 2020. Biomedical entity representations with synonym marginalization. In $A C L$.

George Tsatsaronis, Michael Schroeder, Georgios Paliouras, Yannis Almirantis, Ion Androutsopoulos, Eric Gaussier, Patrick Gallinari, Thierry Artieres, Michael R Alvers, Matthias Zschunke, et al. 2012. Bioasq: A challenge on large-scale biomedical semantic indexing and question answering. In 2012 AAAI Fall Symposium Series.

Ellen Voorhees, Tasmeer Alam, Steven Bedrick, Dina Demner-Fushman, William R Hersh, Kyle Lo, Kirk Roberts, Ian Soboroff, and Lucy Lu Wang. 2020. Trec-covid: Constructing a pandemic information retrieval test collection. arXiv preprint arXiv:2005.04474.

Lucy Lu Wang, Kyle Lo, Yoganand Chandrasekhar, Russell Reas, Jiangjiang Yang, Darrin Eide, Kathryn Funk, Rodney Kinney, Ziyang Liu, William Merrill, et al. 2020. Cord-19: The covid-19 open research dataset. arXiv preprint arXiv:2004.10706.

Jerry Wei, Chengyu Huang, Soroush Vosoughi, and Jason Wei. 2020. What are people asking about covid19? a question classification dataset. arXiv preprint arXiv:2005.12522.

Zhilin Yang, Junjie Hu, Ruslan Salakhutdinov, and William Cohen. 2017. Semi-supervised qa with generative domain-adaptive nets. In $A C L$.

Wonjin Yoon, Jinhyuk Lee, Donghyeon Kim, Minbyul Jeong, and Jaewoo Kang. 2019. Pre-trained language model for biomedical question answering. arXiv preprint arXiv:1909.08229.

Edwin Zhang, Nikhil Gupta, Rodrigo Nogueira, Kyunghyun Cho, and Jimmy Lin. 2020. Rapidly deploying a neural search engine for the covid-19 open research dataset: Preliminary thoughts and lessons learned. arXiv preprint arXiv:2004.05125. 